\title{
Crosstalk between gut microbiota and Sirtuin-3 in colonic inflammation and tumorigenesis
}

\author{
Yong Zhang ${ }^{1}$, Xiao-lan Wang ${ }^{1}$, Min Zhou ${ }^{1}$, Chao Kang ${ }^{1}$, He-dong Lang ${ }^{1}$, Meng-ting Chen ${ }^{1}$, Suo-cheng Hui ${ }^{1}$, \\ Bin Wang ${ }^{1}$ and Man-tian $\mathrm{Mi}^{1}$
}

\begin{abstract}
Colorectal cancer (CRC) is a disease involving a variety of genetic and environmental factors. Sirtuin-3 (Sirt3) is expressed at a low level in cancer tissues of CRC, but it is unclear how Sirt3 modulates colonic tumorigenesis. In this study, we found that gut microbiota play a central role in the resistance to CRC tumor formation in wild-type (WT) mice through APC (Adenomatous Polyposis Coli)-mutant mouse microbiota transfer via Wnt signaling. We also found that Sirt3-deficient mice were hypersusceptible to colonic inflammation and tumor development through altered intestinal integrity and p38 signaling, respectively. Furthermore, susceptibility to colorectal tumorigenesis was aggravated by initial commensal microbiota deletion via Wnt signaling. Mice with Sirt3-deficient microbiota transfer followed by chemically induced colon tumorigenesis had low Sirt3 expression compared to WT control microbiome transfer, mainly due to a decrease in Escherichia/Shigella, as well as an increase in Lactobacillus reuteri and Lactobacillus taiwanensis. Collectively, our data revealed that Sirt3 is an anti-inflammatory and tumor-suppressing gene that interacts with the gut microbiota during colon tumorigenesis.
\end{abstract}

\section{Introduction}

Colorectal cancer (CRC) is a complex multifactorial disease with different incidences around the world ${ }^{1}$. Both genetic and environmental factors contribute to the pathogenesis of $\mathrm{CRC}$, and a series of gene-environment interactions have been shown to contribute to disease susceptibility ${ }^{2,3}$. In recent years, with the development of high-throughput sequencing technology, the gut microbiome has emerged as a central environmental factor in CRC progression ${ }^{4-7}$. However, only a few CRC-related gene-environment interactions have been found, and it remains unclear how host genes interact with the gut microbiome.

\footnotetext{
Correspondence: M.-t. Mi (mi_mt2009@hotmail.com)

${ }^{1}$ Research Center for Nutrition and Food Safety, Institute of Military Preventive Medicine, Third Military Medical University; Chongqing Key Laboratory of Nutrition and Food safety; Chongqing Medical Nutrition Research Center, Chongqing, China
}

Sirtuin-3 (Sirt3), an $\mathrm{NAD}^{+}$-dependent deacetylase, plays a role in metabolism regulation and energy balance. Recent studies have highlighted the importance of mitochondrial Sirt3 in the regulation of $\mathrm{ROS}^{8}$. Tissue-specific deletion of Sirt3 in murine models has shown that Sirt3 performs critical roles in complex diseases, such as metabolic syndrome and diabetes $^{9,10}$. However, the function of Sirt3 in CRC is largely unknown. High expression of the gene encoding Sirt3 is negatively associated with lymph node metastasis and the tumor stage of CRC patients ${ }^{11}$. Sirt3 exerts a tumorsuppressive function in cancer cells ${ }^{12}$. Thus, further exploration of the biological function of Sirt3 in colon tumorigenesis could provide novel strategies for its treatment.

Sirtuins, such as Sirt1, have been linked to altered gut microbiota and susceptibility to colon tumorigenesis ${ }^{13,14}$. The human gut harbors trillions of microbes and plays an important role in the development and progression of 
$\mathrm{CRC}^{15}$. Various studies further support that there are multiple mechanisms by which gut microbiota contribute to CRC tumorigenesis, including the infection of direct enterotoxigenic bacterial pathogens, triggering of the $\mathrm{T}$ helper type-17 inflammatory response, carcinogenic Tolllike receptor signaling and production of microbial oncogenic metabolites ${ }^{16}$.

The aim of this study was to identify the tumorsuppressive function of Sirt3 using Sirt3-deleted murine models with colitis-induced tumorigenesis. Furthermore, we compared the gut microbiota of Sirt3-deficient and WT mice to explore the mechanisms of CRC pathogenesis. Finally, we removed or transferred gut microbiota to reveal host-microbiota interactions in CRC.

\section{Materials and methods Breeding and genotyping}

Sirt3 knockout (SIRT3KO) mice (129/SvlmJ) were purchased from the Jackson Laboratory (Stock Number 012755, Bar Harbor, ME, USA), and male APC min/+ mice (Adenomatous Polyposis Coli,C57BL/6J) were obtained from the Model Animal Research Center of Nanjing University (Nanjing, China). Female WT mice (129/SvlmJ) were crossed with male SIRT3KO mice to generate the heterozygous females or hemizygous males, which were subsequently crossed to generate male Sirt3-/mice and littermates of Sirt3 $+/+$ mice. Age-matched male WT $(129 / \mathrm{Svlm}$ J or C57BL/6J) mice were used for fecal microbiota transplant by initial microbiota removal using an antibiotic cocktail for 3 weeks. The antibiotic treatment was administered via drinking water and consisted of a mixture of $0.2 \mathrm{~g} \mathrm{~L}^{-1}$ ampicillin, $0.1 \mathrm{~g} \mathrm{~L}^{-1}$ vancomycin, $0.2 \mathrm{~g} \mathrm{~L}^{-1}$ neomycin and $0.2 \mathrm{~g} \mathrm{~L}^{-1}$ metronidazole. The mice were maintained under specific pathogen-free (SPF) conditions in an animal experimental center at the Third Military Medical University (Chongqing, China). The protocol was approved by the Animal Care and Use Committee of the Third Military Medical University. Genotyping was done by routine PCR assays on tail DNA using an animal tissue DNA extraction kit and master mix (Tiangen, Beijing, China). The specific primers used to characterize the WT, knockout or mutant mice are listed in Supplemental Table S1.

\section{Experimental design}

In the initial experiment, male SIRT3KO or WT littermates (8 weeks old) were subjected to dextran sulfate sodium (DSS)-induced colitis by adding DSS (MW 36-40 $\mathrm{kDa}, \mathrm{MP}$ Biologicals) to drinking water at a $2.5 \%$ weight volume for 6 days (Supplementary Figure 1). In the gene susceptibility cancer experiment, SIRT3KO mice and WT mice were exposed to an AOM/DSS colon tumor cycle and four DSS cycles, which is one additional cycle than previously described ${ }^{17}$. In the microbiota susceptibility cancer experiment, WT mice and WT mice colonized with 8-week-old male APC $\mathrm{min} /+$ mice microbiota (multiple antibiotics for 3 weeks plus 2 week microbiota adjustment) were exposed to AOM/DSS colon tumor cycles. In the gut commensal microbiota cancer protection experiment, SIRT3KO mice and SIRT3KO mice receiving multiple antibiotics (from week 7 to week 10) were treated by AOM/DSS cycles as mentioned above. In the gene-microbiota interaction experiment, male C57BL/6J mice first received multiple antibiotics for 3 weeks, and the fecal microbiota of 10-week-old male SIRT3KO or WT littermate mice were then transferred for 2 weeks (once a week) according to a previous study ${ }^{18}$. Freshly obtained feces were diluted $1 / 100(\mathrm{wt} / \mathrm{vol})$ in sterile $0.01 \mathrm{M}$ phosphate-buffered saline (PBS, pH 7.4), vortexed for $2 \mathrm{~min}$, and centrifuged for $5 \mathrm{~min}$ at $400 \times g$. The upper suspensions were obtained and washed with PBS three times, and bacterial suspension was used for intragastric administration. Finally, microbiotatransferred C57BL/6J mice were exposed to AOM/DSS colon tumor cycles.

\section{Quantification of cytokines}

Colonic homogenates were obtained by a tissue homogenizer (IKA, Germany). Colonic myeloperoxidase (MPO) activity (Abcam, Inc.) and serum cytokine levels, including IL-1 $\beta$ (R\&D Systems, Inc.), TNF- $\alpha$ (BioLegend, Inc.) and IL-6 (BioLegend, Inc.), were quantified by ELISA.

\section{Western blot analysis}

Proteins were extracted from colon tissues using tissue lysis buffer (Thermo Scientific) supplemented with a protease inhibitor cocktail (Roche Diagnostics). The samples were resolved in 10-15\% SDS-PAGE and transferred onto PVDF membranes. The membranes were blocked in $5 \%$ nonfat milk for $1 \mathrm{~h}$, and primary antibodies were incubated with the membranes separately under rotation overnight at $4{ }^{\circ} \mathrm{C}$. The membranes were incubated with HRP-conjugated secondary antibody for $1 \mathrm{~h}$, and the proteins were visualized using a chemoluminescence system (FUSION, France) with Millipore Immobilon ECL substrate (Millipore, Inc.). The primary antibodies were Caspase-3 (1:1000 dilution, Beyotime, China), $\beta$-catenin (1:1000 dilution, Beyotime), HIF-1 $\alpha$ (1:1000, 3716, Beyotime, China), p38 (1:1000, Abcam), Claudin 8 (1:1000, Abcam), CLCN3 (1:1000, Abcam), Foxo3 (1:1000, Abcam), ERK (1:1000, Abcam), JNK (1:1000, Abcam), CLIC4 (1:1000, Abcam), CLCN4 (1:1000, Sigma), SLC26A2 (1:1000, Sigma), Sirt3 (1:1000, Cell Signaling), phospho-p53 (1:1000, Cell Signaling), GSK-3 $\beta$ (1:1000, Santa Cruz), Claudin15 (1:1000, Santa Cruz), Occludin (1:1000, Santa Cruz) and $\beta$-actin (1:1000, Bestbio, China). 
Histological evaluation and immunofluorescence staining

Mouse colonic tissues were fixed in $10 \%$ neutral formalin followed by gradient dehydration. The tissues were then embedded in paraffin and sectioned at $5 \mu \mathrm{m}$ thickness. The sectioned slides were stained with hematoxylineosin (HE) for microscopic assessment (Olympus, Tokyo, Japan). Histological score was evaluated blindly according to a previous research with minor modification ${ }^{19}$. Briefly, inflammation severity, ulceration and hyperplasia account for 30,30 , and $40 \%$, respectively. Each field was divided into 5 grade levels $(0,1,2,3$, and 4$)$.

For immunofluorescence staining, colon sections were first treated with an antigen-retrieval solution (Beyotime) for $5 \mathrm{~min}$ followed by a wash with by phosphate-buffered saline (PBS) containing $0.1 \%$ Triton X-100 for $10 \mathrm{~min}$. The sections were then blocked with $20 \%$ rabbit serum for $1 \mathrm{~h}$. After incubation with primary antibody against Claudin 15 or ERK at $4{ }^{\circ} \mathrm{C}$ overnight, the samples were incubated for $30 \mathrm{~min}$ with appropriate FITC- or Cy3labelled secondary antibodies (Invitrogen, Thermo Scientific) at room temperature for $1 \mathrm{~h}$. The nuclei were stained with DAPI at a concentration of $100 \mathrm{~g} \mathrm{~mL}^{-1}$. The slides were then examined with a Leica confocal microscope (Leica Inc., Germany), and images were analyzed with LAS Lite (Leica Inc., Germany).

\section{Mouse fecal occult blood tests}

Fecal occult blood tests were performed using freshly collected feces with a commercial kit (Nanjing Jiancheng Bioengineering Institute, Nanjing). Briefly, o-toluene solution and hydrogen peroxide were mixed with mouse feces, and the color changes were observed. The results were classified by the following 5 levels: solution turned blue within $3 \mathrm{~min}$ ( 0 , negative), $2 \mathrm{~min}$ (1, weak positive), 30-60 s (2, positive), blue immediately (3), and dark blue immediately (4, strongly positive).

\section{5 rRNA gene sequencing}

DNA from stool and intestinal contents (QIAamp DNA Stool Mini Kit, Germany) was amplified using barcoded V3-V4 region primers targeting the bacterial 16S rRNA gene, sequenced using the Illumina MiSeq platform (Illumina, San Diego, CA, USA), analyzed using QIIME, and assessed by multiple parameters with STAMP software. For third-generation sequencing, purified fecal DNA was amplified using bacterial 16S rRNA full-length primers and sequenced using Pacbio RSII system (Pacific Biosciences, USA). Principal coordinates analysis (PCoA) was performed using R software.

\section{Fluorescence in situ hybridization (FISH) with bacteria}

Specific oligonucleotide probes were synthesized according to previous studies with the primers listed in Supplemental Table $\mathrm{S2}^{20,21}$. Microbial FISH was performed as previously described ${ }^{22}$. Colon sections were fixed in $10 \%$ neutral formalin for $10 \mathrm{~min}$, and they were then treated with $1 \mathrm{mg} \mathrm{mL}^{-1}$ lysozyme in $100 \mathrm{mM}$ Tris- $\mathrm{HCl}(\mathrm{pH}$ 7.2) for $10 \mathrm{~min}$ at room temperature. The sections were subsequently washed in PBS buffer $(\mathrm{pH} 7.2)$ and equilibrated in a FISH solution $(900 \mathrm{mM} \mathrm{NaCl}, 20$ $\mathrm{mM}$ Tris- $\mathrm{HCl}[\mathrm{pH} 8.0], 0.01 \%$ sodium dodecyl sulfate, and $30 \%$ formamide). FISH was performed on slides overnight at $55^{\circ} \mathrm{C}$ in a humidity chamber with $150 \mu \mathrm{L}$ of FISH solution containing $4 \mu \mathrm{l}$ of probe $(50 \mathrm{ng} / \mu \mathrm{l})$, reaching a final concentration of $4 \mathrm{ng} \mu \mathrm{L}^{-1}$. Finally, DAPI was added to the wash solution to a final concentration of 100 $n g \mathrm{~mL}^{-1}$ for staining the nuclei.

\section{Results}

\section{Sirt3 diminishes susceptibility to Colitis}

SIRT3KO mice subjected to DSS exhibited exacerbated colonic shortening $(p<0.01$, Fig. 1a) and increased inflammatory cytokine levels compared to WT mice (MPO, IL- $1 \beta$ and TNF- $\alpha ; p<0.05$, Fig. 1b). SIRT3KO + DSS mice displayed increased histological damage in the mucosal integrity of large intestine compared to WT mice $(p<0.05$, Fig. 1c). The leaky gut phenotype is characterized by altered expression of tight junction components, including Occludin, Claudin8 and Claudin $15^{23}$. Consistently, colons from SIRT3KO+DSS mice had decreased gene expression of Occludin, Claudin 8 and Claudin 15 compared to WT mice (Fig. 1d), demonstrating a deficit in intestinal barrier integrity. Consistent with the genotyping results, the colonic expression of Sirt3 significantly decreased in SIRT3KO+DSS mice compared to WT mice (Fig. 1d). Most notably, phosphorylated STAT3 was increased in SIRT3KO+DSS mice compared to WT mice (Fig. 1d). Immunofluorescence staining also demonstrated a low Claudin15 level in SIRT3KO+DSS colons (Fig. 1e). Additionally, SIRT3KO+DSS mice consistently exhibited increased levels of Bacteroides/Firmicutes compared to WT mice (Fig. 1f). PCoA analysis of 16S rRNA gene sequencing showed an alteration of the microbiome profiling in the colonic content in SIRT3KO+DSS mice (Fig. 1g). Furthermore, the microbial communities of Alloprevotella and an unclassified genus from the Gastranaerophilales order were significantly higher in SIRT3KO+DSS mice than in WT mice $(P<0.05$, Fig. $1 \mathrm{~h})$. Collectively, these results identify a critical role for Sirt3 in actively regulating mucosal barrier function and gut homeostasis in mice.

\section{Resistance of WT mice to Colitis-associated cancer is diminished by APC min+/- mice microbiome transfer}

WT (129/SvlmJ) mice subjected to AOM/DSS tumorigenesis exhibited resistance to colonic tumor formation, while WT mice colonized with adult APC min $+/-$ mice microbiota developed a significantly increased number of 


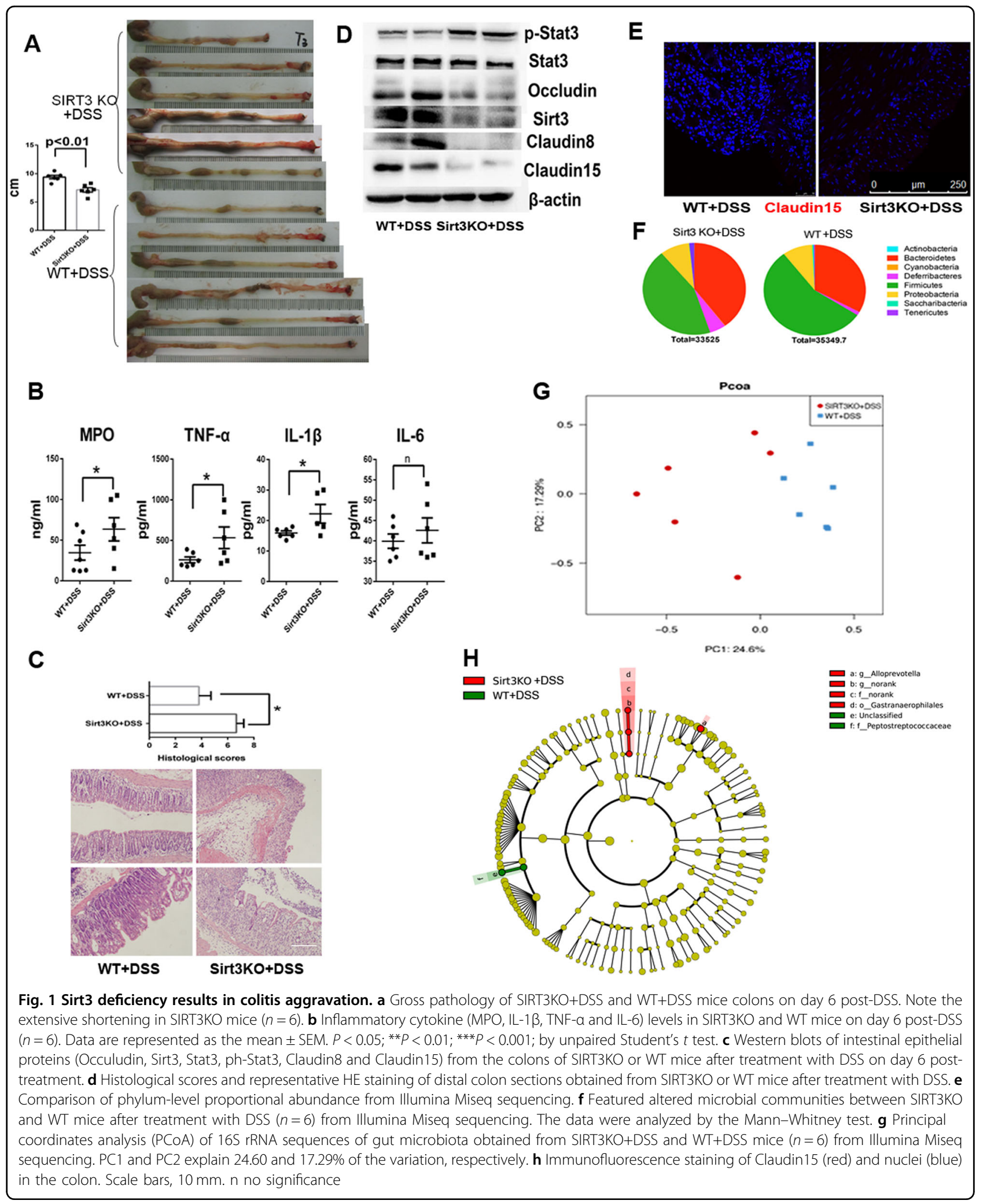

intestinal polyps $(P<0.001$, Fig. 2a, b) and more histological tumor burden in the large intestine $(P<0.01$, Fig. 2c). Chloride ion-dependent $C L C N 3$ has been shown to prevent colon tumorigenesis in a previous study ${ }^{24}$. In the present study, there was no apparent difference of colonic CLCN3 expression in APC FTp mice and WT 
A

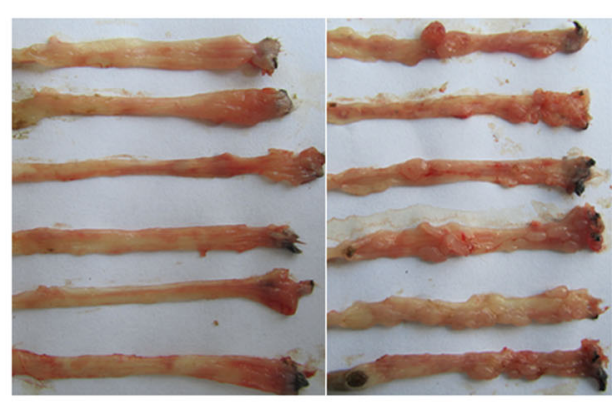

WT

APC FTp
C
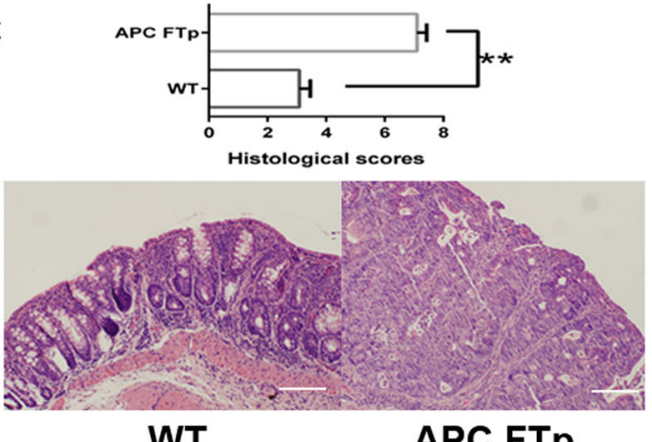

WT

APC FTp

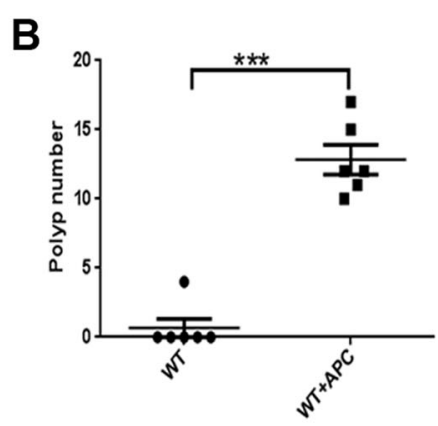

D

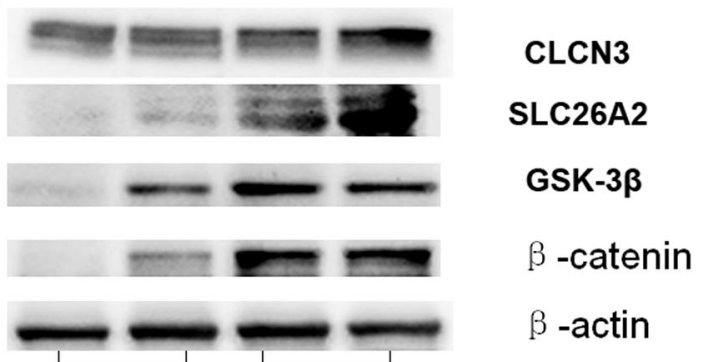

WT APC FTp

E

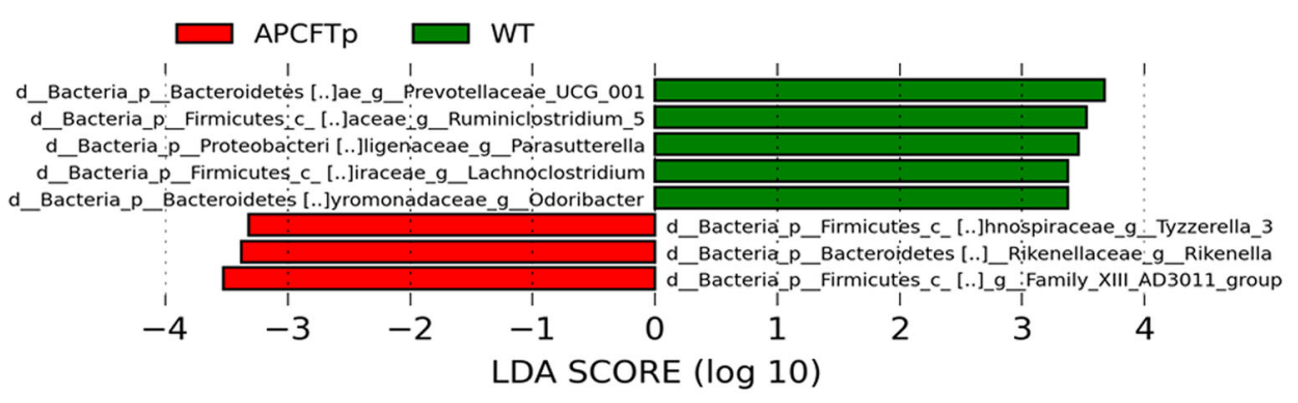

F

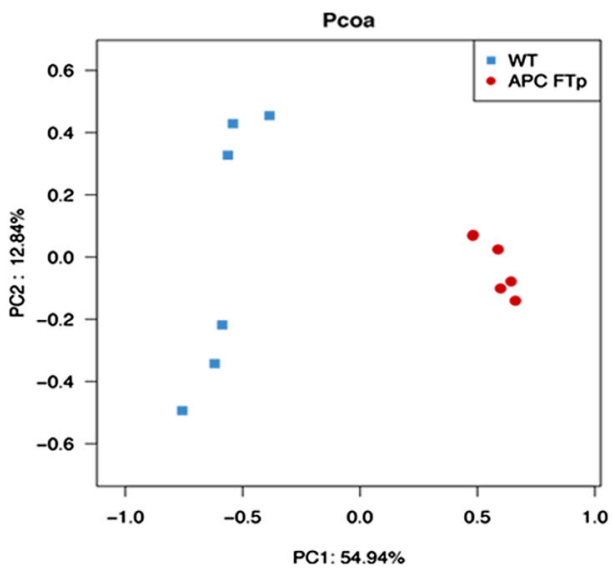

Fig. 2 Microbiota modify the host genotype to colon cancer. a Images of the distal colon from APC FTp and WT mice after AOM/DSS tumorigenesis. $\mathbf{b}$ Comparison of polyp numbers between APC FTp and WT mice $(n=6)$. Data are represented as the mean \pm SEM. $P<0.05$; ${ }^{* *} P<0.01$; ${ }^{* * *} P<0.001$; by unpaired Student's $t$ test. $\mathbf{c}$ Histological scores and representative HE staining of distal colon sections obtained from APC FTp and WT mice after AOM/DSS tumorigenesis. d Western blot of intestinal CLCN3, SLC26A2, GSK3 $\beta$ and $\beta$-catenin expression from APC FTp or WT mice after AOM/DSS tumorigenesis. e Featured altered microbial communities between APC FTp and WT mice after AOM/DSS tumorigenesis $(n=6)$ from Illumina Miseq sequencing. Data were analyzed by the Mann-Whitney test. f PCoA of 165 rRNA sequences of gut microbiota obtained from the APC FTp and WT mice $(n=6)$ from Illumina Miseq sequencing. PC1 and PC2 explain 54.94 and $12.84 \%$ of the variation, respectively. $n$ no significance 
mice (Fig. 2d), but the expression of SLC26A2, which contributes to the intestinal exchange of chloride and sulfate, was increased in the colon of APC FTp mice and influenced the sulfate transport. Moreover, colons from APC FTp mice showed increased gene expression of GSK3 $\beta$ and $\beta$-catenin compared to WT mice (Fig. $2 \mathrm{~d}$ ), demonstrating an activation of the Wnt signaling pathway. With regard to the gut microbiome analysis, WT mice harbored high levels of Prevotellaceae UCG-001, Lachnoclostridium, Odoribacter, Ruminiclostridium and Parasuttella, while APC FTp mice harbored high levels of an unknown genus belonging to Family XIII-AD3011, Rikenella and Tyzzerella bacteria (Fig. 2e). Above the family level, APC FTp mice harbored increased levels of Peptococcaceae and Actinobacteria, as well as a decreased level of Gamma-proteobacteria (Fig. S2). There was also a significant difference in the microbiome profiling between APC FTp mice and WT mice (Fig. 2f). Collectively, these results indicated a key role for the gut microbiome in promoting susceptibility to colon cancer in mice.

\section{Sirt3 knockout mice are hypersusceptible to colonic tumor development}

To evaluate the tumor-suppressive role of Sirt3, SIRT3KO and WT mice were subjected to AOM/DSS tumorigenesis (Fig. S3). As expected, SIRT3KO mice showed increased polyp numbers and an increased severity of colonic proliferation compared to WT mice (Fig. 3a-d), both of which suggest a tumor-suppressive role of Sirt3. Previous in vitro cancer cell studies have shown that Sirt3 regulates HIF- $1 \alpha$ to suppress ROS and reduces intracellular $\mathrm{NAD}^{+}$levels to repress Erk1/2 and upregulate p53 as well as activation of caspase- 3 to induce cell death ${ }^{12}$. We investigated the expression of p-p53, HIF1- $\alpha$, ERK, Caspase 3 and p38 in the colon carcinogenesis of SIRT3KO and WT mice. SIRT3KO mice showed decreased expression of HIF1- $\alpha$, ERK and Caspase3 but increased intestinal expression of $\mathrm{p} 38$ and Claudin 15 compared to WT mice (Fig. 3e, f). We also found that SIRT3KO mice had lower liver ERK expression (Fig. 3g). CLCN4 has been implicated in the development of colon cancer, and higher CLCN4 expression has been observed in Sirt3-deficient mice (Fig. 3f $)^{25}$. Consistently, the Sirt3 target protein, FOXO3a, was downregulated in Sirt3-deficient mice (Fig. 3f). Microbiome analysis revealed that Escherichia/Shigella and Lactococcus were more abundant in SIRT3KO samples than WT samples $(P<0.05$, Fig. 3 h). There was also a significant difference in the microbiome profiling between SIRT3KO and WT mice (Fig. 3i).

\section{Presence of commensal microbiota reduces the incidence of colon tumors}

To determine if the presence of commensal microbiota influences AOM/DSS tumorigenesis, we pre-treated
SIRT3KO mice (genotyping by Fig. S3) with a broadspectrum antibiotic cocktail (vancomycin, neomycin, ampicillin and metronidazole) for initial microbiota removal and then subjected them to AOM/DSS cycles. As a result, the incidence of tumors increased, and mucosal bleeding was enhanced in antibiotic-treated mice (Fig. 4a-c). Evaluation of key bacterial taxa that discriminate SIRT3KO mice from antibiotic-treated mice revealed that the presence of commensal microbiota significantly altered the levels of several OTUs (Fig. 4d). Specifically, the genus Alistipes was more abundant in SIRT3KO mice, while an uncultured genus (belonging to the Peptococcaceae family) and genus Lachnospiraceae UCG-001 were more abundant in SIRT3KO+Abs mice (Fig. 4d). Intestinal bleeding was promoted in SIRT3KO + Abs mice as indicated by occult blood tests (Fig. 4e). Compared to SIRT3KO mice, SIRT3KO+Abs mice showed enhanced intestinal CLCN4, GSK3 $\beta$ and $\beta$ catenin expression but not JNK and CLIC4 expression (Fig. 4f). There was also a significant difference in the microbiome profiling between SIRT3KO and SIRT3KO +Abs mice (Fig. 4g). Additionally, SIRT3KO mice exhibited a gut tumor-probiotic effect with some beneficial bacterial taxa, such as Coriobacterium and Lactobacillus, which were enriched on tumor samples (Fig. 4h).

\section{Transmitted SIRT3 expression by the microbiome determines susceptibility to colon cancer}

To identify the SIRT3-related gut microbes playing a key role in the development of colonic tumorigenesis, we transferred the gut microbiome of SIRT3KO mice into antibiotic-treated C57BL/6J mice in comparison with WT microbiome transfer. As expected, SIRT3KO TP mice were more susceptible to AOM/DSS tumorigenesis and developed more tumors than WT TP mice $(P<0.05$, Fig. 5a, b). Moreover, the adiponectin level was significantly higher in WT TP mice than in SIRT3KO TP mice $(P<0.05$, Fig. $5 c)$. Microbiota transfer also impacted intestinal SIRT3, GSK3 $\beta, \quad \beta$-catenin and Claudin15 expression (Fig. 5d). Compared to WT TP mice, SIRT3KO TP mice consistently exhibited decreased levels of Firmicutes/Bacteroides and a higher proportion of Proteobacteria and Verrucomicrobia (Fig. 5e). Furthermore, an altered gut microbiome was observed with Escherichia/Shigella dysenteriae more abundant in SIRT3KO TP mice and Lactobacillus reuteri, Lactobacillus taiwanensis and Helicobacter mesocricetorum more abundant in WT TP mice $(P<0.05$, Fig. 5f). These data suggested that Escherichia/S. dysenteriae, well-known infective pathogens, may play a role in colonic tumorigenesis but that Lactobacillus reuteri and Lactobacillus taiwanensis may exert a protective effect. $H$. mesocricetorum is a commensal rodent bacterium. The SIRT3KO TP mouse samples clustered distinctly from WT TP mice 
A

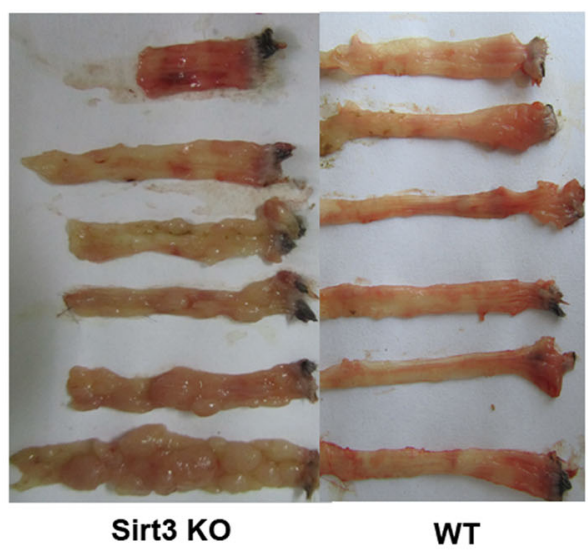

C

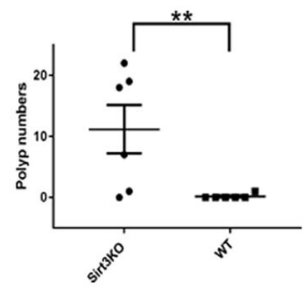

D

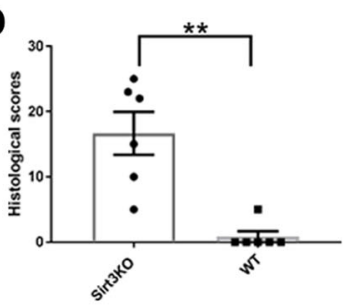

F

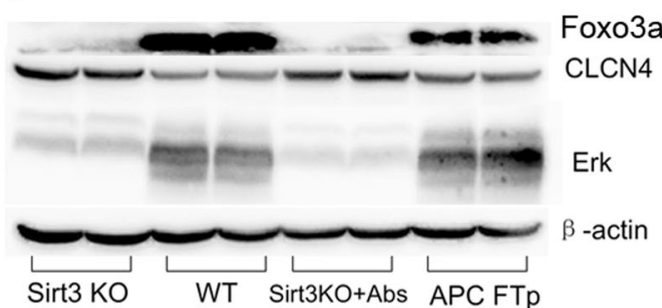

H

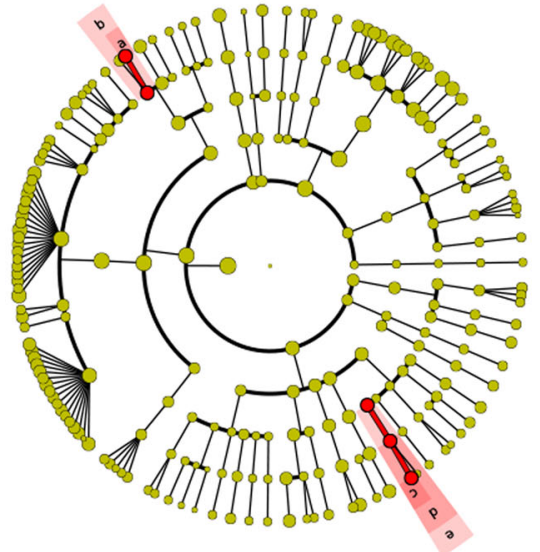

B

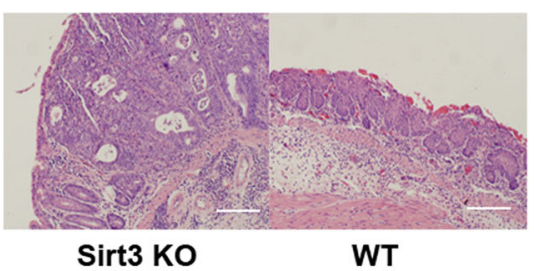

E

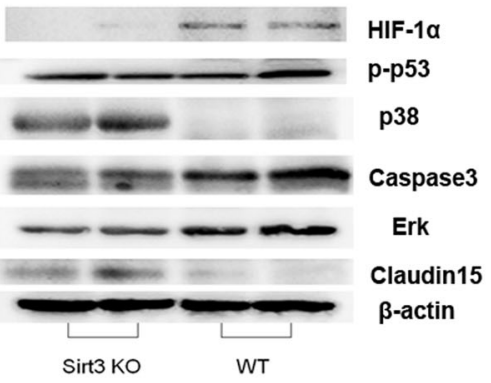

G

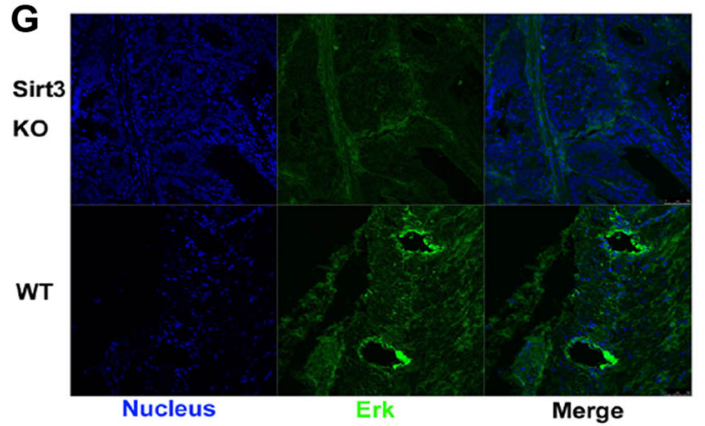

Nucleus

Merge

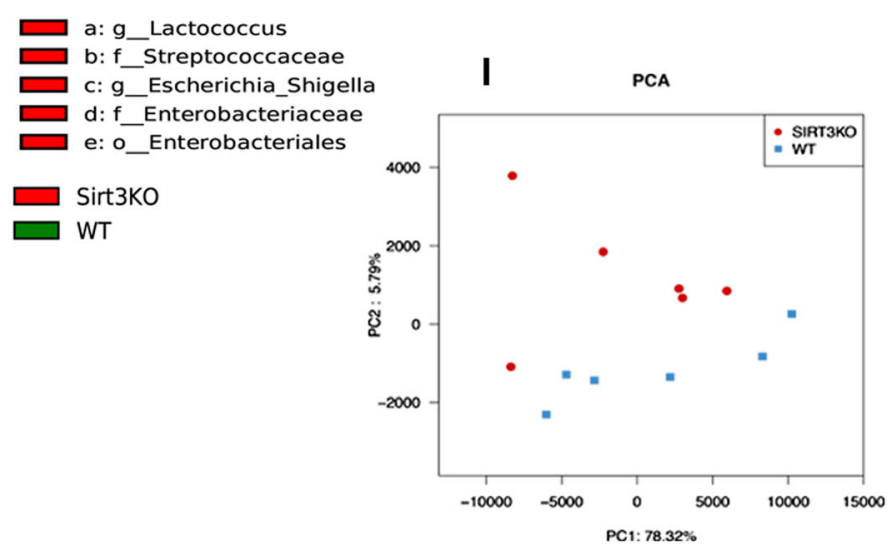

Fig. 3 Sirt3 deficiency results in increased susceptibility to colonic tumors. a Images of distal colons from SIRT3KO and WT mice after AOM/DSS tumorigenesis. $\mathbf{b}$ Representative HE staining of distal colon sections obtained from SIRT3KO and WT mice after AOM/DSS tumorigenesis. c Comparison of polyp numbers between SIRT3KO and WT mice $(n=6)$. Data are represented as the mean \pm SEM. $P<0.05 ;{ }^{* *} P<0.01$; ${ }^{* * *} P<0.001$; by unpaired Student's $t$ test. $\mathbf{d}$ Histological scores of distal colon sections from SIRT3KO and WT mice after AOM/DSS tumorigenesis $(n=6)$. e Western blots of intestinal HIF1-a, Erk, p-p53, p38, Claudin 15 and caspase3 expression from SIRT3KO and WT mice after AOM/DSS tumorigenesis. f Western blots of liver CLCN4 and Erk expression from SIRT3KO and WT mice after AOM/DSS tumorigenesis. $\mathbf{g}$ Immunofluorescence staining of Erk (green) and nuclei (blue) in colons. Scale bars, $10 \mathrm{~mm}$. $\mathbf{h}$ Featured altered microbial communities between SIRT3KO and WT mice after AOM/DSS tumorigenesis $(n=6)$ from Illumina Miseq sequencing. Data were analyzed by the Mann-Whitney test. i PCoA of $16 \mathrm{~S}$ rRNA gene-sequencing analysis of gut microbes obtained from the indicated mice and treatments $(n=6)$ from Illumina Miseq sequencing. PC1 and PC2 explain 78.32 and $5.79 \%$ of the variation, respectively. $\mathrm{n}$ no significance 
A

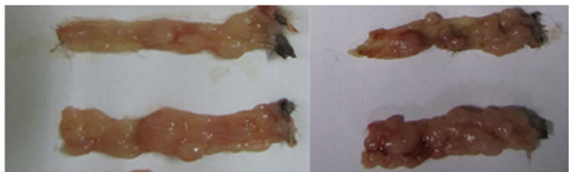

Sirt3Ko

D

口irt3KO

d_Bacteria_P_Firmicutes_c_t...)Peptocọccaceae_g_uncultured

d_Bacteria_P_Firmicutes_c. (..)e_g_Lachnospiraceà_UCG_001

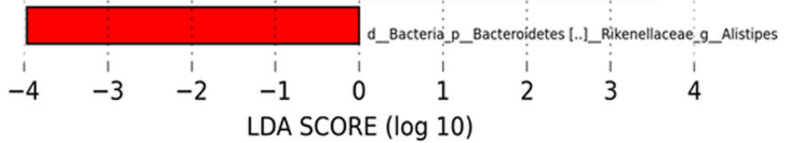

E

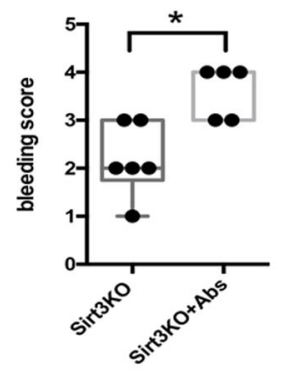

F

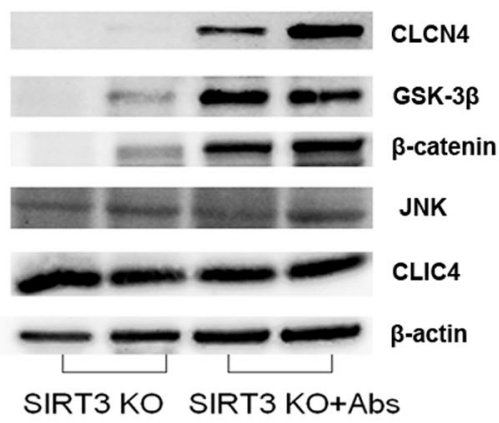

C
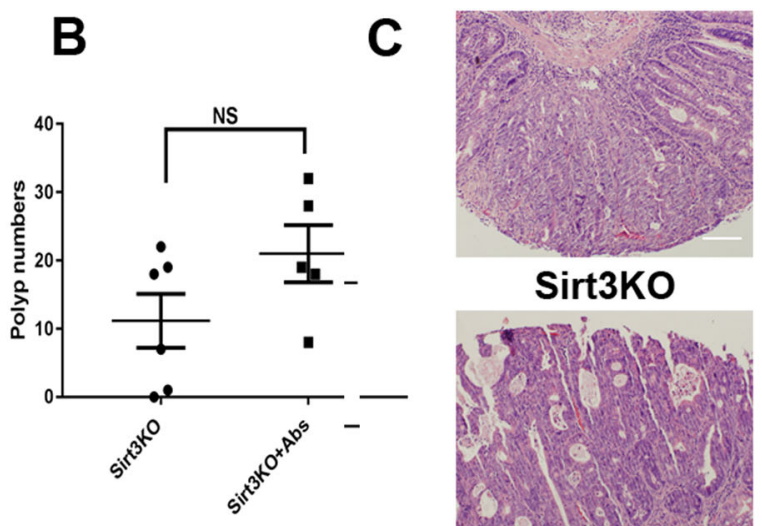

Sirt3KO

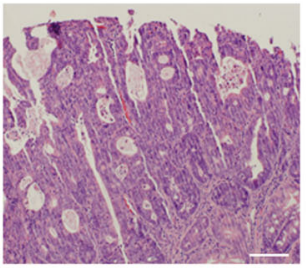

Sirt3KO+Abs

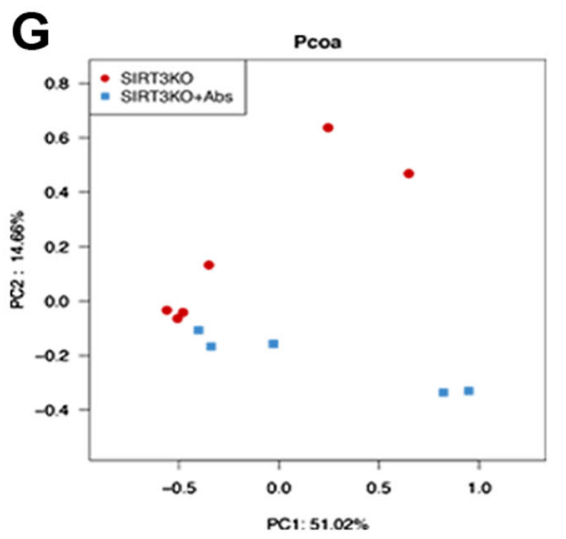

H Nucleus Coriobacterium Lactobacillus Merge Sirt3Ko

\section{Sirt3KO+Abs}

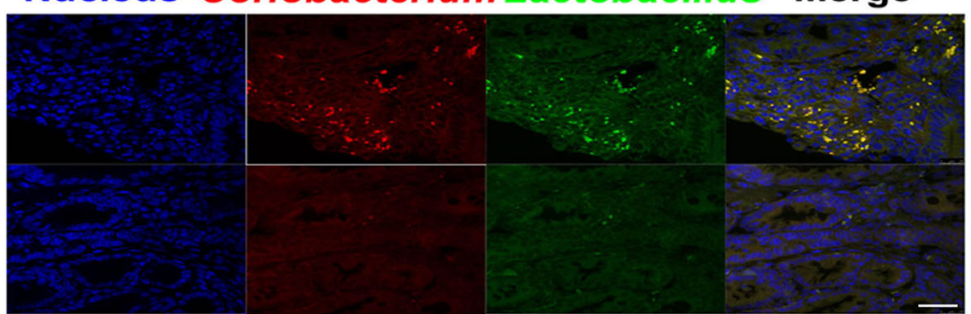

Fig. 4 Commensal microbiota protect against severe colon cancer in SIRT3KO mice. a Images of distal colons from SIRT3KO and SIRT3KO+Abs mice after AOM/DSS tumorigenesis. $\mathbf{b}$ Comparison of polyp numbers between SIRT3KO and SIRT3KO+Abs mice $(n=5-6)$. The data are represented as the mean \pm SEM. $P<0.05 ;{ }^{* *} P<0.01 ;{ }^{* * *} P<0.001$; by unpaired Student's $t$ test. $\mathbf{c}$ Representative HE staining of distal colon sections obtained from SIRT3KO and SIRT3KO+Abs mice after AOM/DSS tumorigenesis. $\mathbf{d}$ Featured altered microbial communities between SIRT3KO and SIRT3KO+Abs mice after AOM/DSS tumorigenesis $(n=5-6)$ from Illumina Miseq sequencing. Data were analyzed by the Mann-Whitney test. e Fecal occult blood tests of SIRT3KO and SIRT3KO+Abs mice $(n=5-6)$. $\mathbf{f}$ PCOA of $16 \mathrm{~S}$ rRNA gene-sequencing analysis of gut microbes obtained from the SIRT3KO and SIRT3KO +Abs mice $(n=5-6)$ from Illumina Miseq sequencing. PC1 and PC2 explain 51.02 and $14.66 \%$ of the variation, respectively. $\mathbf{g}$ Western blots of intestinal CLCN4, JNK, CLIC4, GSK3 $\beta$ and $\beta$-catenin expression from SIRT3KO and SIRT3KO+Abs mice after AOM/DSS tumorigenesis. $\mathbf{h}$ FISH with Lactobacillus (green) and Coriobacterium (red) from distal colons of SIRT3KO and WT mice after AOM/DSS tumorigenesis. n no significance 


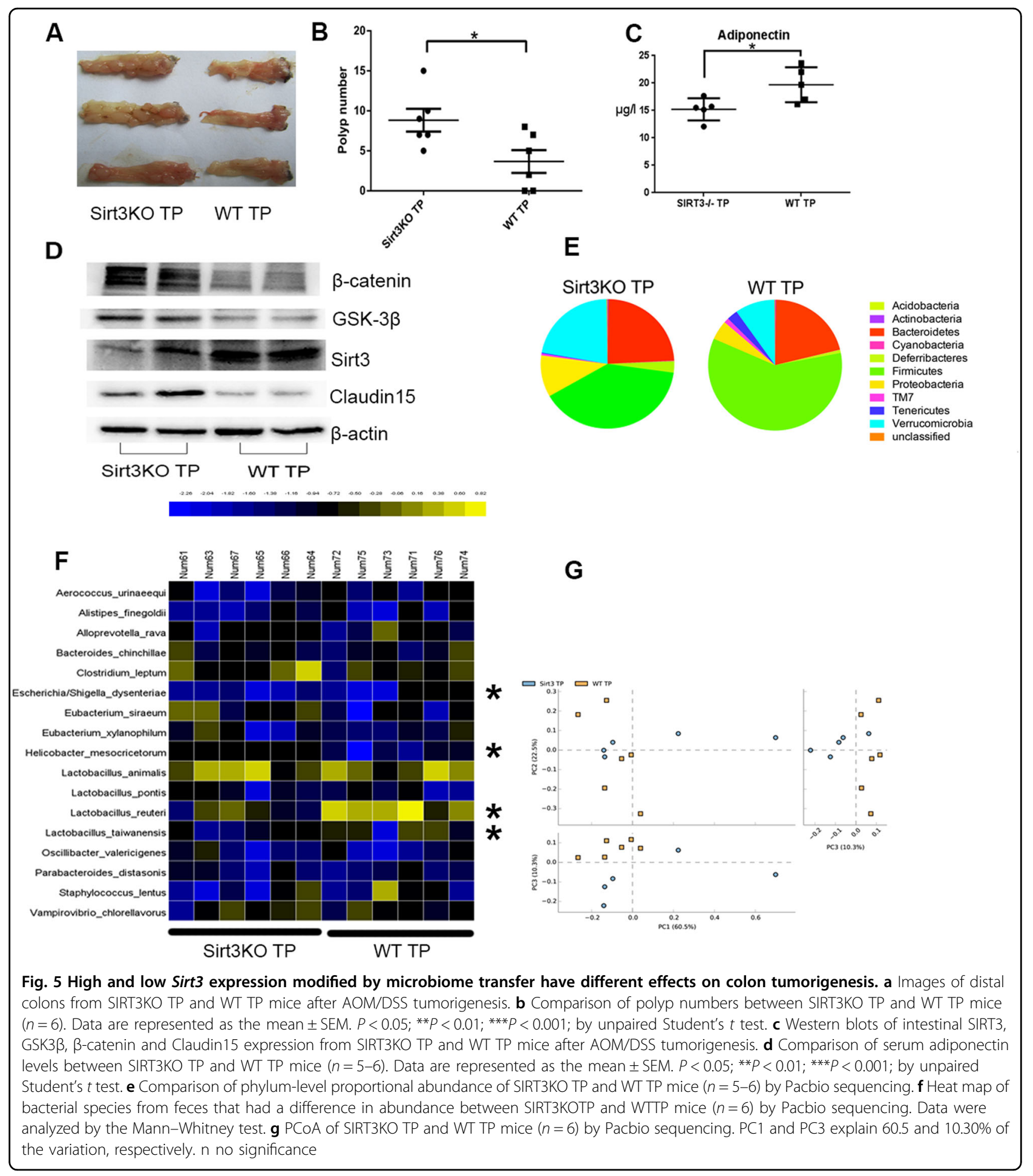

by $\mathrm{PCoA}(\mathrm{PC} 1=60.5 \%$ and $\mathrm{PC} 3=10.3 \%)$, which also indicated different gut microbiome when Sirt3 was depleted (Fig. 5g). Finally, we assessed if Sirt3 activity was influenced by the gut microbiome, and we found that mice transplanted with SIRT3KO samples had low Sirt3 expression (Fig. 5c).

\section{Discussion}

It is well established that chronic inflammation of the colon can promote the incidence of carcinogenesis ${ }^{26,27}$. A clinical study also supported the idea that inflammation is an independent risk factor for colon neoplasia formation in IBD patients ${ }^{28}$. In this study, we aimed to uncover the 
potential role of Sirt3 in the regulation of intestinal barrier integrity and inflammation. In this study, mice lacking Sirt3 exhibited a higher inflammatory level, increased intestinal epithelial damage, and an altered gut microbiome compared to WT mice. Furthermore, Sirt3 deficiency induced altered expression of intestinal epithelial proteins, especially Claudin15. Previous research has shown that the expression of epithelial Claudin 15 is positively associated with a significant deficit in intestinal barrier function ${ }^{23}$. We hypothesized that loss of Sirt3 would thus influence intestinal barrier integrity and increase susceptibility to colitis via Claudin 15 and the STAT3 pathway.

Despite affecting the intestinal barrier integrity, Sirt3 is a multifunctional tumor suppressor that influences the expression of $\mathrm{p} 38$ and Caspase 3 but not HIF1- $\alpha$, ERK and $\mathrm{p} 53^{12}$. A recent study demonstrated that $\mathrm{p} 38$ is required to maintain metabolism in CRC and that p38 inhibition can result in FOXO3 activation followed by caspase- 8 and HER3 upregulation, thereby resulting in ERK1/2 overactivation in vivo ${ }^{29}$. To our knowledge, FOXO3, a direct target of Sirt3, can protect against oxidative damage ${ }^{30}$. These results indicate that the crosstalk between the ERK1/ 2 and p38 signaling plays a key role in Sirt 3 susceptibility to $\mathrm{CRC}$ and that Sirt3 mainly inhibits intestinal p38 signaling as well as suppresses tumor growth and development. Here we identified an unexpected result for Sirt3 that differs from its known role in Claudin15 signaling. A possible explanation for the opposite expression of Claudin15 in colitis and colitis-associated colon cancer is that the colon of CRC is in a hyperproliferative state.

Previous research has found that Fusobacterium nucleatum-enriched intestinal cancer tissue with activated $\beta$ catenin signaling potentiates tumorigenesis in a mouse model $^{31}$. Similar observations were made in our study. First, the transfer of the fecal microbiome of APC-mutant mice to WT mice eliminated resistance to AOM/DSS tumorigenesis with the activation of GSK3 $\beta / \beta$-catenin. In contrast, high abundance of Rikenella might participate in sulfated mucin degradation, activate SLC26A2 expression and regulate intestinal sulfate transport with sulfated mucin desulfatase activity ${ }^{32}$. Hydrogen sulfide, a sulfatereducing product, may induce DNA damage and play a role in colon carcinogenesis ${ }^{33}$. Moreover, the presence of commensal microbiota delayed the deterioration of gut carcinogenesis and suppressed $\beta$-catenin signaling. Previous research has reported that commensal microbiota induce a late onset of proinflammatory and protumorigenic responses after $\mathrm{AOM} / \mathrm{DSS}^{34}$. The commensal microbiota were mainly characterized by a high abundance of Alistipes, which has been demonstrated as a potential probiotic at higher levels in tumor-bearing mice ${ }^{35}$. Additionally, a high abundance of Peptococcaceae has also been reported in tumor-bearing mice. Consistently, a recent study also reported that Peptostreptococcus anaerobius is detected with higher abundance in CRC and promotes mouse colorectal tumorigenesis by inducing intracellular cholesterol biosynthesis ${ }^{36}$.

Finally, transfer of Sirt3-deficient fecal microbiota to C57BL/6 mice exhibited aggravated inflammationassociated colon tumorigenesis due to a high abundance of potentially infective Escherichia/Shigella dysenteriae and low abundance of beneficial L. reuteri and L. taiwanensis. A previous study demonstrated that Escherichia/ Shigella dysenteriae may promote p38 MAP kinase activation $^{37}$. Lactobacillus spp., which exerts an inhibitory effect on Escherichia/Shigella in vitro and in vivo, has been demonstrated to have an anti-tumor effect, including apoptotic, antioxidant and immune-regulating functions $^{38}$. A recent study revealed that $L$. reuteri and $L$. taiwanensis stimulate aryl hydrocarbon receptor (AHR) activity to reduce colitis and regulate gut microbiota ${ }^{39}$. In summary, the mechanism driving microbiome-linked colonic tumorigenesis may involve the p38 and GSK3 $\beta /$ $\beta$-catenin signaling pathways.

For the first time, we showed a Sirt3-microbiome interaction, of which C57BL/6 recipients colonized with the microbiome from SIRT3KO mice had decreased Sirt3 expression in comparison with WT microbiota transfer. The key microbes that prevent downregulation of Sirt3 expression during colorectal tumorigenesis are $L$. reuteri and $L$. taiwanensis, which may provide a precise therapeutic strategy for the prevention of Sirt3-deficient colon cancer by manipulation of the gut microbiome. Moreover, Sirt3 and commensal microbiota synergize to regulate the expression of CLCN4 in the development of colon cancer. CLCN4 has been shown to enhance colon cancer migration and metastasis. CLCN4 has been proposed as a risk factor for colon cancer, but its role in the early stages of colon cancer is unknown ${ }^{25}$. In summary, CLCN4 is critical for Sirt3-microbiome coordination with a tumorsuppressing effect, but further study is needed.

Considering the gut probiotic effect of SIRT3KO mice, it is possible that probiotic bacteria, such as Lactobacillus, may provide bacterial Sir2 (a gene was homologous to eukaryotic Sirt3) for deacetylase activity. A previous in vitro experiment demonstrated that bacterial Sir2 activity is elevated with co-culture of Lactobacillus acidophilus and Caco-2 cells ${ }^{40}$. Thus, Sirt3 may be crucial for a probiotic effect in gut tumorigenesis. In conclusion, Sirt3 is a novel colonic tumor suppressor that inhibits colonic tumorigenesis via an interaction with the gut microbiome.

\section{Accession numbers}

The original Miseq and Pacbio sequences of the 16S profiling have been deposited to a BioProject (PRJNA341959) in NCBI and are available under accession numbers SRS1736448-SRS1751469. 


\section{Acknowledgements}

This study was supported by grants from the Chongqing Postdoctoral Research Project (Xm2014125).

\section{Authors contributions:}

Y.Z. and M.-t.M. designed the experiments; Y.Z., M.Z., and M.-t.C. performed the experiments: C.K., X.-I.W. and Y.Z. analyzed the data; C.K. and X.Z. contributed to the western blot experiment and bioinformatics analysis; B.W. and X.II.W. contributed reagents and materials; Y.Z. and M.-t.M. wrote the paper; and H.-d.L. and S.-c.H. commented on the manuscript.

\section{Conflict of interest}

The authors declare that they have no conflict of interest.

\section{Publisher's note}

Springer Nature remains neutral with regard to jurisdictional claims inpublished maps and institutional affiliations.

Supplementary information accompanies this paper at https://doi.org/ 10.1038/s12276-017-0002-0.

Received: 2 August 2017 Revised: 19 October 2017 Accepted: 24 October 2017.

Published online: 13 April 2018

\section{References}

1. Torre, L. A. et al. Global cancer statistics, 2012. Ca. Cancer J. Clin. 65, 87-108 (2015).

2. Haggar, F. A. \& Boushey, R. P. Colorectal cancer epidemiology: incidence, mortality, survival, and risk factors. Clin. Colon Rectal Surg. 22 191-197 (2009)

3. Kantor, E. D. et al. Gene-environment interaction involving recently identified colorectal cancer susceptibility loci. Cancer Epidemiol. Biomark. Prev. 23 1824-1833 (2014).

4. Wang, T. et al. Structural segregation of gut microbiota between colorectal cancer patients and healthy volunteers. ISME J. 6, 320-329 (2012).

5. Zeller, G. et al. Potential of fecal microbiota for early-stage detection of colorectal cancer. Mol. Syst. Biol. 10, 766 (2014).

6. Feng, Q. et al. Gut microbiome development along the colorectal adenomacarcinoma sequence. Nat. Commun. 6, 6528 (2015).

7. Kasai, C. et al. Comparison of human gut microbiota in control subjects and patients with colorectal carcinoma in adenoma: Terminal restriction fragment length polymorphism and next-generation sequencing analyses. Oncol. Rep. 35, 325-333 (2016).

8. Bause, A. S. \& Haigis, M. C. SIRT3 regulation of mitochondrial oxidative stress. Exp. Gerontol. 48, 634-639 (2013).

9. Hirschey, M. D. et al. SIRT3 deficiency and mitochondrial protein hyperacetylation accelerate the development of the metabolic syndrome. Mol. Cell. 44, 177-190 (2011).

10. Lantier, L. et al. SIRT3 is Crucial for maintaining skeletal muscle insulin action and protects against severe insulin resistance in high-fat-fed mice. Diabetes $\mathbf{6 4}$ 3081-3092 (2015).

11. Liu, C., Huang, Z., Jiang, H. \& Shi, F. The Sirtuin 3 expression profile is associated with pathological and clinical outcomes in colon cancer patients. Biomed. Res Int 2014, 871263 (2014).

12. Chen, Y. et al. Sirtuin-3 (SIRT3), a therapeutic target with oncogenic and tumorsuppressive function in cancer. Cell Death Dis. 5, e1047 (2014).

13. Lakhan, S. E. \& Kirchgessner, A. Gut microbiota and sirtuins in obesity-related inflammation and bowel dysfunction. J. Transl. Med. 9, 202 (2011).

14. Lo Sasso, G. et al. Loss of Sirt1 function improves intestinal anti-bacterial defense and protects from colitis-induced colorectal cancer. PLOS ONE $\mathbf{9}$, e102495 (2014).

15. Louis, P., Hold, G. L. \& Flint, H. J. The gut microbiota, bacterial metabolites and colorectal cancer. Nat. Rev. Microbiol. 12, 661-672 (2014).
16. Chen, H. M. et al. Decreased dietary fiber intake and structural alteration of gut microbiota in patients with advanced colorectal adenoma. Am. J. Clin. Nutr. 97, 1044-1052 (2013).

17. Neufert, C., Becker, C. \& Neurath, M. F. An inducible mouse model of colon carcinogenesis for the analysis of sporadic and inflammation-driven tumor progression. Nat. Protoc. 2, 1998-2004 (2007).

18. Zheng, P. et al. Gut microbiome remodeling induces depressive-like behaviors through a pathway mediated by the host's metabolism. Mol. Psychiatry 21, 786-796 (2016).

19. Man, S. M. et al. Critical role for the DNA sensor aim2 in stem cell proliferation and cancer. Cell 162, 45-58 (2015).

20. Harmsen, H. J. et al. Development of 165 rRNA-based probes for the Coriobacterium group and the Atopobium cluster and their application for enumeration of Coriobacteriaceae in human feces from volunteers of different age groups. Appl. Environ. Microbiol. 66, 4523-4527 (2000).

21. Quevedo, B. et al. Phylogenetic group- and species-specific oligonucleotide probes for single-cell detection of lactic acid bacteria in oral biofilms. BMC Microbiol. 11, 14 (2011).

22. Mueller, S. et al. Differences in fecal microbiota in different European study populations in relation to age, gender, and country: a cross-sectional study. Appl. Environ. Microbiol. 72, 1027-1033 (2006).

23. Hsiao, E. Y. et al. Microbiota modulate behavioral and physiological abnormalities associated with neurodevelopmental disorders. Cell 155, 1451-1463 (2013).

24. Zhang, Y. et al. Lactobacillus casei Zhang and vitamin K2 prevent intestinal tumorigenesis in mice via adiponectin-elevated different signaling pathways. Oncotarget 8, 24719-24727 (2017).

25. Ishiguro, T. et al. Gene trapping identifies chloride channel 4 as a novel inducer of colon cancer cell migration, invasion and metastases. Br. J. Cancer 102, 774-782 (2010).

26. Jess, T., Rungoe, C. \& Peyrin-Biroulet, L. Risk of colorectal cancer in patients with ulcerative colitis: a meta-analysis of population-based cohort studies. Clin. Gastroenterol. Hepatol. 10, 639-645 (2012).

27. Beaugerie, L. \& Itzkowitz, S. H. Cancers complicating inflammatory bowel disease. N. Engl. J. Med. 372, 1441-1452 (2015).

28. Rutter, M. et al. Severity of inflammation is a risk factor for colorectal neoplasia in ulcerative colitis. Gastroenterology 126, 451-459 (2004).

29. Chiacchiera, F. et al. Blocking p38/ERK crosstalk affects colorectal cancer growth by inducing apoptosis in vitro and in preclinical mouse models. Cancer Lett. 324, 98-108 (2012).

30. Tseng, A. H., Shieh, S. S. \& Wang, D. L. SIRT3 deacetylates FOXO3 to protect mitochondria against oxidative damage. Free. Radic. Biol. Med. 63, 222-234 (2013).

31. Kostic, A. D. et al. Fusobacterium nucleatum potentiates intestinal tumorigenesis and modulates the tumor-immune microenvironment. Cell Host. Microbe 14, 207-215 (2013).

32. Bomar, L., Maltz, M., Colston, S. \& Graf, J. Directed culturing of microorganisms using metatranscriptomics. MBio 2, e00012 (2011).

33. Attene-Ramos, M. S., Wagner, E. D., Gaskins, H. R. \& Plewa, M. J. Hydrogen sulfide induces direct radical-associated DNA damage. Mol. Cancer Res. 5, 455-459 (2007)

34. Zhan, Y. et al. Gut microbiota protects against gastrointestinal tumorigenesis caused by epithelial injury. Cancer Res. 73, 7199-7210 (2013).

35. Borges-Canha, M., Portela-Cidade, J. P., Dinis-Ribeiro, M., Leite-Moreira, A. F. \& Pimentel-Nunes, P. Role of colonic microbiota in colorectal carcinogenesis: a systematic review. Rev. Esp. Enferm. Dig. 107, 659-671 (2015).

36. Tsoi, H. et al. Peptostreptococcus anaerobius induces intracellular cholesterol biosynthesis in colon cells to induce proliferation and causes dysplasia in mice. Gastroenterology 152, 1419-1433 (2017).

37. Lautz, K. et al. NLRP10 enhances Shigella-induced pro-inflammatory responses. Cell. Microbiol. 14, 1568-1583 (2012).

38. Zhong, L., Zhang, X. \& Covasa, M. Emerging roles of lactic acid bacteria in protection against colorectal cancer. World J. Gastroenterol. 20, 7878-7886 (2014).

39. Lamas, B. et al. CARD9 impacts colitis by altering gut microbiota metabolism of tryptophan into aryl hydrocarbon receptor ligands. Nat. Med. 22, 598-605 (2016).

40. Yanagihara, S., Hirota, T. \& Yamamoto, N. Transcriptional response of Lactobacillus acidophilus L-92 after attachment to epithelial Caco-2 cells. J. Biosci. Bioeng. 114, 582-585 (2012). 